\title{
Internal Medicine Point of Care Ultrasound: Indicators It's Here to Stay
}

\author{
Mike Wagner, MD, FACP, RDMS ${ }^{1,2}$, Keith R. Barron, $M D^{3,4}$, and Renee Dversdal, \\ $M D, F A C P^{5}$
}

'Department of Internal Medicine, Prisma Health-Upstate, Greenville, USA; ${ }^{2}$ University of South Carolina School of Medicine Greenville, Greenville, USA; ${ }^{3}$ Department of Internal Medicine, University of South Carolina School of Medicine, Columbia, USA; ${ }^{4}$ Prisma Health-Midlands, Columbia, USA; ${ }^{5}$ Oregon Health \& Science University, Portland, USA.

J Gen Intern Med 34(10): 1956-58

DOI: $10.1007 / \mathrm{s} 11606-019-05268-0$

(c) Society of General Internal Medicine 2019

$\mathrm{W}$ e congratulate our Canadian colleagues on their latest publication advancing the field of Internal Medicine Point-of-Care Ultrasound (IMPOCUS) ${ }^{1}$. The authors, members of the Canadian Internal Medicine UltraSound Group (CIMUS), are building a strong foundation for widespread implementation of IMPOCUS curricula across Canada. The group first published an expert-based consensus framework for Internal Medicine (IM) residency training programs in $2017^{2}$, and in this edition of The Journal, they describe the rationale and process for identifying education indicators for IMPOCUS curriculum development efforts. The CIMUS Group reached a consensus on 22 indicators covering four categories: teaching, learning environment and program organization, data management and quality assurance, and trainee and program assessments. These indicators are a clever way to monitor IMPOCUS programs as they grow but also guide them along the right path to help ensure consistent highquality IMPOCUS education across the country. More data are very much needed and we look forward to their group further developing and tracking these indicators as they help light the way ahead.

What is the current state of IMPOCUS in the USA? As the authors noted there are only 17 Canadian IM residency programs compared with over 400 in America, making consensus and widespread implementation a challenge for any subject, POCUS or otherwise. While some institutions like Abbott Northwestern, Scripps Mercy, and the University of South Carolina have emphasized IMPOCUS in their curricula for over a decade, a 2017 survey found that only $37 \%$ of IM residencies had a formal IMPOCUS curriculum ${ }^{3}$, up from $25 \%$ in $2012^{4}$. IMPOCUS education is slowly expanding; recently four IM residency programs published a curricular development experience piece that serves as a basic guide for programs wishing to start an ultrasound training program ${ }^{5}$.

Published online August 19, 2019
In the meantime, new IM trainees are increasingly coming to residency with exposure to ultrasound training during medical school. They are frequently expected to perform POCUS while on Emergency Medicine (EM) and Critical Care (CC) rotations, as POCUS is considered a core competency for EM residency training ${ }^{6}$ and is an entrustable professional activity for CC fellowships ${ }^{7}$. Rare IMPOCUS trained faculty often must share their knowledge during patient care or volunteer time on the side. While total hours of faculty time spent teaching IMPOCUS can be trended as an education indicator, inclusion of faculty protected time or FTE dedicated to IMPOCUS would help capture and clarify support from the program, not just faculty enthusiasm. With limited access to IMPOCUS faculty, eager residents often learn independently or from each other, leading to a "hidden curriculum." Their perception, whether correct or not, is often that POCUS improves their exam and therefore patient care, regardless of their training, supervision, or setting. Findings may not be documented in the record and images not saved, but still might influence thought processes and management decisions. For some, this "phantom scanning" practice justifiably raises concerns.

The use of POCUS, with or without formal training, is about to expand on a much larger scale with the advent of powerful, pocket-sized devices that are priced affordably to be leased or purchased by the individual. Entering into the era of ubiquitous personal ultrasounds presents an imperative. In the academic IM community, we need to ask ourselves: is the majority status quo of marginalizing or outsourcing POCUS training in the best interest of our specialty and our patients?

In addition to the work by the CIMUS group, there are now several "indicators" IMPOCUS is stepping out of the shadows as a legitimate academic field within IM:

1. Position statements from relevant IM societies taking a supportive stance on POCUS.

The Society of Hospital Medicine has recently published numerous position statements on POCUS for guided procedures as well as for diagnostic use. The American College of Physicians has formally acknowledged the important role of IMPOCUS, has created an IMPOCUS Advisory Group, and is currently working on additional curricular offerings. The 
Society of General Internal Medicine leadership Council has placed POCUS as a top priority for its Clinical Practice Committee, and a statement from the Alliance for Academic Internal Medicine recognizing and supporting the integration of POCUS across the longitudinal training environment was accepted and is pending publication.

2. Representation of IMPOCUS within international ultrasound organizations and collaboration with IM organizations.

The American Institute of Ultrasound in Medicine (AIUM), World Interactive Network Focused on Critical Ultrasound (WINFOCUS), and Society for Ultrasound in Medical Education (SUSME) are leading multidisciplinary umbrella organizations, all of which include general internists in leadership positions, support IMPOCUS courses, and offer educational resources.

3. Increasing IMPOCUS educational offerings at the national level.

IMPOCUS panels, courses, workshops, and online content have been offered at every major IM meeting in the past year. Based on popularity and overwhelmingly positive feedback, these can be expected to increase in coming years.

\section{Development of IMPOCUS fellowships.}

Dedicated fellowships in IMPOCUS have expanded from one in $2011^{8}$ to four in 2019. Some Academic GIM Fellowships have offered IMPOCUS as a research focus, and several EM fellowships now accept IM trainees. The rapid growth of ultrasound fellowships helped provide significant expertise to meet the demand for Ultrasound Director positions within EM and generated much of the EMPOCUS literature.

A pathway for ultrasound curriculum development and implementation is well-charted in the field of EM, the current leaders in POCUS education. Other factors that were essential to the EMPOCUS movement some 20 years ago ${ }^{9}$ should be considered for IM.

- Inclusion of POCUS images and concepts on in-training and board certification/recertification exams.

- Announcement by the Residency Review Committee (RRC) that training in POCUS is considered mandatory for residents.

- Research and scholarly work by POCUS users regularly presented at academic specialty meetings and published in specialty journals.

For better or worse, board exams drive medical curricula to a large degree. MKSAP includes POCUS content and inclusion on formal examinations could help advance POCUS curricula out of necessity. While the majority of IM programs are not prepared for an IMPOCUS training mandate, a statement by the RRC similar to that for critical care, that proficiency in POCUS is "strongly recommended," would help IM programs better prepare for this level of commitment.
IMPOCUS is still underrepresented in IM literature and meetings compared with EM and CC. Recruiting a pool of reviewers with POCUS expertise for POCUS-related submissions, sponsoring special editions on POCUS, as in the July 2018 Edition of Southern Medical Journal, or recurring POCUS segments such as in Chest's "POCUS Corner" may facilitate awareness and acceptance within the IM community.

Implementation of IMPOCUS can and should follow a similar path as EMPOCUS, but like EM almost 30 years ago, it is evident that we must take an active role in charting our own course. The CIMUS group reached consensus to exclude tracking indicators of external support from both radiology and cardiology. While we certainly should work with and draw upon the extensive experience of other specialties, we will need to define POCUS for our specialty rather than let it be defined for us. To address POCUS across departments, hospitals and medical systems are developing system-wide policies on image archiving and electronic health record integration. Internists should engage in this process to ensure they can be adapted to hospitalist and outpatient workflows. Our training and practice cover a wide range of settings, and our patients have a broad spectrum of disease severity that may affect POCUS interpretation and utility. Many practical and philosophical questions remain to be answered. As the largest medical specialty, the widespread use of this technology in IM will have a tremendous impact on the medical system. Our perspective matters.

A key concept from the article by Ma et al. is POCUS as a disruptive technology. The thought that POCUS is poised to change something as fundamental as the way physicians assess their patients may be unsettling for some internists, particularly when a large part of our professional identity is being physical exam experts. But we must empower ourselves with evidence-based tools that aid diagnosis at the bedside as they become available if we also want to maintain our identity of master diagnosticians. For assessing our IM patients with multiple complex medical problems, POCUS is an excellent addition to the clinical encounter to help put the pieces of the puzzle together. A core multisystem ultrasound exam, suitable for both the inpatient and outpatient settings, can be performed in less than 3 minutes while still preserving much of the art and "feel" of a traditional exam ${ }^{10}$. We must continue to evolve and move forward to embrace IMPOCUS as a core clinical skill. We agree with the authors when they say we must "ensure that POCUS program development is deployed in a thoughtful manner and with broad support"; the focus is no longer "why" but "how?" To successfully integrate and expand the field of IMPOCUS to its full potential in our specialty, a massive collective effort will be necessary. It will be difficult. But we are internists. We are problem solvers. And we are up for this challenge. 
Corresponding Author: Mike Wagner, MD, FACP, RDMS; Department of Internal MedicinePrisma Health-Upstate, Greenville, USA (e-mail: mike.wagner@prismahealth.org).

\section{Compliance with Ethical Standards:}

Conflict of Interest: The authors declare no relevant conflicts of interest.

\section{REFERENCES}

1. Ambasta, A., Balan, M., Mayette, M. et al. J Gen Intern Med (2019). https://doi.org/10.1007/s11606-019-05124-1

2. Ma IWY, Arishenkoff $\mathbf{S}$, Wiseman $\mathbf{J}$, et al. Internal Medicine Point-ofCare Ultrasound Curriculum: Consensus Recommendations from the Canadian Internal Medicine Ultrasound (CIMUS) Group. J Gen Intern Med. 2017;32(9): 1052-57.

3. Reaume $\mathbf{M}$, Siuba $\mathbf{M}$, Wagner $\mathbf{M}$, et al. Prevalence and Scope of Point-ofCare Ultrasound Education in Internal Medicine, Pediatric, and Medicine-Pediatric Residency Programs in the United States. J Ultrasound Med. 2019 Jun;38(6):1433-1439.
4. Schnobrich DJ, Gladding S, Olson APJ, et al. Point-of-care ultrasound in internal medicine: a national survey of educational leadership. J Grad Med Educ. 2013;5(3):498-502.

5. LoPresti CM, Schnobrich DJ, Dversdal RK, et al. A road map for pointof-care ultrasound training in internal medicine residency. Ultrasound J. 2019 Dec; 11(10).

6. Ultrasound Guidelines: Emergency, Point-of-Care and Clinical Ultrasound Guidelines in Medicine. Ann Emerg Med. 2017;69(5):e27-e54.

7. Fessler HE, Addrizzo-Harris D, Beck JM, et al. Entrustable professional activities and curricular milestones for fellowship training in pulmonary and critical care medicine: executive summary from the Multi-Society Working Group. Crit Care Med. 2014;42(10):2290-2291

8. Barron KR, Wagner MS, Hunt PS, et al. A Primary Care Ultrasound Fellowship: Training for Clinical Practice and Future Educators. J Ultrasound Med. 2019 Apr;38(4):1061-1068.

9. Heller MB, Mandavia D, Tayal Vs, et al. Residency training in emergency ultrasound: fulfilling the mandate. Acad Emerg Med. 2002 Aug;9(8):835-9.

10. Wagner M, Boughton J. PEARLS for an Ultrasound Physical and Its Routine Use as Part of the Clinical Examination. South Med J. 2018 Jul;111(7):389-394.

Publisher's Note Springer Nature remains neutral with regard to jurisdictional claims in published maps and institutional affiliations. 